\title{
CLASES SOCIALES EN LA COMUNICACIÓN Y EN LA CONFIGURACIÓN DE ETNICIDADES: UNA OMISIÓN EN EL ANÁLISIS DE LA REALIDAD INDOAMERICANA
}

\author{
Héctor Muñoz Cruz ${ }^{1}$ (D)
}

RESUMEN: La sociolingüística en América Latina es una ciencia que se ocupa de las reivindicaciones socioculturales indoamericanas, que implican la desigualdad social en lenguas y culturas comunicativas. En este trabajo se esboza un panorama sobre la desigualdad y la estratificación en la funcionalidad de las lenguas históricas indígenas, haciendo una distinción entre experiencias o discusiones políticas sobre poblaciones indígenas, a modo de historia externa, y la constitución científica de la desigualdad social en el lenguaje como objeto de la lingüística.

Palabras clave: Desigualdad de clases sociales. Lengua y comunicación. Etnicidad. Sociolinguística.

\section{Social classes in communication and in the configuration of ethnicities: An omission in the Analysis of Indo-American Reality}

ABSTRACT: Sociolinguistics in Latin America is a science that deals with the sociocultural claims of the Indo-Americans, which imply social inequality in communicative languages and

\footnotetext{
${ }^{1}$ Universidad Autónoma Metropolitana - Ciudad de México (DF), México. E-mails: hmc@xanum.uam.mx; hmunozcruz@gmail.com

DOI: 10.1590/CC0101-32622019216705
} 
cultures. In this work, a panorama is drawn about inequality and stratification in the functionality of indigenous historical languages, making a distinction between experiences or political discussions on indigenous populations, in the form of external hi tory, and the scientific constitution of social inequality in language as an object of linguistics.

Keywords: Social classes inequality. Language and communication. Ethnicity. Sociolinguistics.

\section{PRELIMINARES}

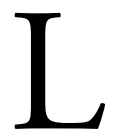

a sociolingüística en América Latina es una ciencia social que se ha ocupado de las reivindicaciones socioculturales indoamericanas desde los años 70 , pese a que no ha podido consolidar un desarrollo científico propio y, especialmente, a que no ha hecho efectivo aun el compromiso sociopolítico que proclamó en sus inicios ${ }^{1}$. Los principales referentes de formación profesional y de investigación de esta disciplina en la región latinoamericana apuntan hacia problemáticas convergentes con esa vocación, tales como la situación subalterna de los pueblos indoamericanos, las crisis y rezagos en educación escolar, la compleja y exponencial migración interna y transnacional y, de manera muy subordinada, la desigualdad social y cultural en materia de lenguaje; más específicamente, las consecuencias de las diversidades étnicas y las estratificaciones sociales de clase en las interacciones y actividades comunicativas.

La escasa importancia atribuida en el programa científico de esta disciplina a las discusiones sobre las interrelaciones entre las lenguas históricas copresentes en las sociedades actuales con las etnodiversidades y las estratificaciones de clases sociales representa un claro indicio de que no se han acumulado suficientes conocimientos propios sobre la naturaleza, estructura e impacto de estos conflictos sociolingüísticos, pero además que no se han aprovechado creativa y críticamente los aportes de las teorías sociales en esta materia. Con todo, esta disciplina goza de difusión y aceptación en los contextos sociales concernidos ${ }^{2}$. 
Es interesante, por otra parte, advertir que son visibles y cotidianas las actividades de describir, regular y evaluar la importancia social de las lenguas y los modos culturales de la comunicación en los diversos dominios de las sociedades, casi sin excepción. Sin embargo, contra lo esperado, un supuesto subyace inmutable en las prácticas valorativas y de regulación que emiten comunidades, ciudadanos e instituciones gubernamentales, supuesto que se resume en las siguientes palabras: es posible intentar diseños de una sociedad multicultural armónica y plurilingüe, sirviéndonos de la lógica de economías capitalistas abiertas, que manejan subsidios y acciones afirmativas para la pobreza, las marginaciones y las desigualdades sociales. Desde esta perspectiva ideológica y de superposición a las estructuras lingüísticas y comunicativas, los discursos institucionales respectivos generan un efecto de espectacularización (LAFONT, 1980), que sugiere la ilusión o la ficción de que existe compatibilidad de intereses entre el Estado, las comunidades de habla y los ciudadanos, respecto del control de los recursos lingüísticos, simbólicos y culturales.

La verdad es que, cuando se trata de estructuras sociales específicas y de condiciones históricas y políticas determinadas, no sirven las declaraciones pluralistas y enfáticas de hablantes, administradores del lenguaje y lingüistas acerca de las desigualdades sociales y etnodiversidades en el lenguaje, porque las soluciones viables y congruentes escapan rápidamente del control de todo mundo.

La complejidad de esta tarea investigativa se dimensiona cuando miramos sus inicios en los ańos sesenta, lo que permite constatar que la sociolingüística ha cambiado su programa científico, sus objetivos y métodos de investigación especialmente. Esto, con la intención de llegar más allá de la mera descripción de la variabilidad, de los cambios de estructura de las lenguas históricas, de la movilidad en la funcionalidad asimétrica y de las interrelaciones hegemónicas en el multilingüismo real, con el loable propósito de interpretar mejor la naturaleza, composición y consecuencias de las transformaciones contemporáneas. En la actualidad, es una disciplina que se ocupa de todos los aspectos de la comunicación verbal en las sociedades humanas. En particular, destaca el análisis empírico detallado sobre cómo las prácticas comunicativas constituyen y reflejan a la vez las relaciones de poder y dominación, también sobre 
cómo el lenguaje influye en la formación y perpetuación de instituciones sociales, así como en la transmisión de la cultura, fenómenos que representan problemas centrales de las ciencias sociales contemporáneas ${ }^{3}$.

De hecho, muchos de los científicos sociales modernos más influyentes conciben los procesos sociales como procesos esencialmente comunicativos. Y sólo necesito mencionar el nombre de M. Ponty, A. Gramsci y, más recientemente, de P. Bourdieu, M. Foucault y el filósofo alemán Habermas, quienes han escrito extensamente acerca de las bases comunicativas de los procesos sociales (GUMPERZ, 1996, p. 33).

Paradójicamente, surge una recurrente incoherencia epistemológica cuando en ámbitos de las ciencias sociales y de instituciones públicas se discute sobre la diversidad lingüística. Se observa que siguen prevaleciendo las interpretaciones abstractas y estructuralistas de las lenguas. La teoría saussureana, que concibe a la gramática como un tópico de análisis independiente y distante de los fenómenos sociales, domina aún en las ciencias sociales y en instituciones que tienen la responsabilidad pública de asesorar a la sociedad, administrando el uso de las lenguas copresentes y fenómenos culturales de la comunicación social. En suma, a pesar de los avances logrados en el estudio de las jerarquías y conflictos socioculturales, la investigación sociolingüística no ha logrado tener influencia en la teoría social ${ }^{4}$, ni ha conseguido un papel orientador independiente en las políticas del lenguaje, que aplican instituciones públicas y privadas. Con cierta ligereza, algunos autores no admiten las incompatibilidades teóricas:

En Sociología, si nos ocupamos, por ejemplo, del estudio de las sociedades de clases, ¿̨por qué no inspirarnos simultáneamente en las lecciones de la lingüística y en las lecciones de la etnología? Vale decir, ¿por qué no proceder análogamente a como procedieron Saussure, Trubetz- koy, Jakobson y Lévi-Strauss? (ROJAS, 1992, p. 18). 
Una y muchas veces, desde la lingüística histórica, la geografía lingüística y algunos enfoques filológicos, se ha demostrado que la variabilidad y cambios en lenguas y formas de comunicación se deben a complejas motivaciones sociales. De hecho, pioneros estudios sociolingüísticos han ofrecido evidencias de que tanto la lengua como el habla reflejan patrones de organización sistemáticos y que operan profundamente relacionados con la división social del trabajo en la sociedad. Hilary Putnam (1975), en particular, desarrolló innovadoras implicaciones semánticas sobre la división social de trabajo, fortaleciendo la concepción de que el conocimiento lingüístico no se desarrolla o proviene de comunidades homogéneas y abstractas, sino que refleja la complejidad y multiplicidad de la cooperación humana interaccional, sobre la cual se posibilita la vida social organizada 5 .

Cada vez es mayor el consenso de que la configuración sociolingüística de una sociedad refleja el andamio social y político de la historia de la diversidad lingüística en una sociedad, y que el impacto regulativo en los hablantes depende de formatos reflexivos sobre comportamientos y normas sociales compartidas por la comunidad de habla. Este andamio sociohistórico y político siempre implica un modelo y una dinámica específica sobre las relaciones sociales y políticas. Hablantes y autores (DOWNES, 1998; CRYSTAL, 2000; MAR-MOLINERO; STEVENSON, 2006; MUÑOZ CRUZ, 2010) son más proclives a reconocer como factores sociales de diferenciación del lenguaje los siguientes:

When we introduced sociolinguistic variables it was mainly in relation to the two interconnected social factores of class and style. This is important but somewhat misleading, since it hioghlights only one particular sociolinguistic structure... The main factors are: (1) geographical space; (2) stratification; (3) social networks; (4) sex; (5) ethnicity; (6) age. These are woven together in a very subtle and complex way in a speech community (DOWNES, 1998, p. 176) ${ }^{6}$.

Desde perspectivas afines, las teorías de Charles S. Peirce (1992), J-F. Dortier (2004) y D. Sperber (2005) han reafirmado la im- 
portancia de la reflexividad sobre los recursos culturales y lingüísticos. Estos autores consolidaron la concepción de la cultura como un proceso que está mediado poderosamente por las representaciones mentales, muchas más direccionadoras e influyentes que las reglas pragmáticas sobre las cuales se materializan verbal y no verbalmente las relaciones sociales cotidianas (PIERCE apud HOUSE; KLOESEL, 1992). A su vez, las relaciones sociales están construidas por los hombres con los materiales que les proporciona la cultura. Y estos materiales, que son por excelencia significativos, tienen su forma privilegiada de expresión en el lenguaje. Así se constituye la realidad simbólica y es así como las relaciones sociales son intercambios de significados entre los hombres (ROJAS, 1992).

En suma, es posible identificar una compleja gama de perspectivas de la investigación sociolingüística, para interpretar mejor el significado comunicativo de las estructuras de clase en contextos indoamericanos. Desafortunadamente, no ha resultado benéfico el acuńamiento acrítico y positivista de conceptos indispensables de las ciencias sociales, tales como "comunidad", "clase social", "sociedad de clase", "identidad étnica”, "conflicto”, "poder”, "dominación”, entre otras, las cuales resultan imprecisas o engañosas como categorías analíticas de los intercambios comunicativos interculturales, por lo que requieren ser replanteadas. Adicionalmente, hay que criticar también la tendencia muy popular de concebir estas pretendidas categorías analíticas como entidades exclusivamente ideológicas, ignorando el hecho de que los procesos sociales implican siempre prácticas comunicativas cotidianas, relaciones culturales globales y también formatos institucionales de regulación sociocultural. Es más, la gravitación y las interacciones entre estas dimensiones de la comunicación social constituyen tal vez el principal reto teórico de las intervenciones sobre la diversidad lingüística y los intercambios interculturales contemporáneos ${ }^{7}$.

Intentaré esbozar en este trabajo un panorama tentativo sobre la desigualdad y la estratificación en la funcionalidad de las lenguas históricas indígenas como resultados "universales" de la existencia de estructuras sociales de clase y de etnodiversidades, valiéndome de un procedimiento práctico. Distinguiré entre coyunturas o discusiones políticas 
relevantes sobre poblaciones indígenas, a modo de historia externa, y aspectos de la constitución científica de las estratificaciones y jerarquías en el uso y status de las lenguas históricas, como episodios significativos de la historia interna de la sociolingüística. La combinación de ambas perspectivas puede mejorar nuestra valoración del reto de la teoría sociolingüística por arraigarse en espacios relevantes de la matriz cultural, étnica y lingüística de Latinoamérica y también por aumentar el reconocimiento en el conjunto de las ciencias sociales ${ }^{8}$.

\section{PERSPECTIVAS POLÍTICAS SOBRE ETNICIDAD Y CLASES SOCIALES EN CONTEXTOS INDÍGENAS}

La historia de las discusiones políticas sobre clases sociales y pueblos indoamericanos ha sido prolongada, cíclica y multifacética. Con un propósito demostrativo, mencionaré ciertas coyunturas sociopolíticas en el continente americano, a modo de casos claros, que nos ayudan a entender la situación desde perspectivas de la economía política y la crítica latinoamericana del capitalismo.

En Estados Unidos, para empezar, una nueva etapa de las interacciones entre las organizaciones indígenas y movimientos de izquierda comienza a desarrollarse, a partir de las protestas en 2017 y las polémicas en torno al apoyo de la confederación sindical AFL-CIO al oleoducto de Dakota (Dakota Access Pipeline - DAPL). Esta circunstancia denota un gran viraje; puesto que en los años ochenta, el Movimiento Indio Americano (American Indian Movement - AIM) manifestaba su claro rechazo a toda la tradición intelectual europea y occidental, afirmando que el marxismo no ofrecía a los pueblos indios de EE.UU. nada mejor que el capitalismo y que concebía a los indígenas y sus tierras como un coste del desarrollo económico (BALTHASER, 2017) ${ }^{9}$.

La historia desigual de las izquierdas y de la soberanía de los pueblos indios de EE.UU. fue abordada ocasionalmente durante el siglo XX. En las primeras décadas del siglo XX, es de hacer notar, los movimientos culturales y políticos pluralistas no lograron sostener sus objetivos de etnicidad y se transformaron o se incorporaron a movimientos anticoloniales y de nacionalismo negro. 
En ese período, se asumía que el marxismo y las luchas indígenas no eran compatibles, debido a que a este teoriza la modernidad capitalista y la transformación histórica, tomando como sujeto al obrero industrial. Jodi Byrd (2015), respecto de la estrategia política marxista del siglo XX, plantea que las teorías de Antonio Gramsci sobre las prácticas contrahegemónicas solo adquieren sentido cuando se piensa en un Estado colonial democrático multiétnico y no en una genuina independencia tribal. En suma, en este contexto el marxismo no estaría considerando los objetivos de soberanía y autodeterminación propias de los pueblos indígenas ${ }^{10}$.

En América Latina, por su parte, el fenómeno de la etnicidad o de la identidad étnica se ha tornado un problema muy relevante en ámbitos de políticas públicas interculturales y medios académicos.

Cabe aclarar, sin embargo, que el empleo de las identidades étnicas para lograr avances políticos no es una estrategia nueva. Como principal fuente de referencia, la Internacional Comunista (Comintern) - a partir de las interpretaciones de Vladimir Lenin sobre nacionalidad y las colonias - defendió los derechos de autodeterminación de las minorías nacionales, incluyendo la separación de las estructuras estatales opresivas $^{11}$, particularmente en la segunda década del siglo XX. Si bien estas discusiones ayudaron a las izquierdas a comprender la situación de las minorías étnicas y el racialismo en los entornos del nacionalismo republicano, se produjeron discrepancias muy profundas en las interpretaciones sobre la lucha de clases (BALTHASER, 2017).

La persistente discusión sobre si la opresión era ante todo un asunto de clase, raza o nacionalidad adquirió una orientación indoamericana en la tercera década del siglo XX. José Carlos Mariátegui postuló en 1929 que la llamada "cuestión indígena" formaba parte de las relaciones de clases, en las cuales la burguesía presionaba a un proletariado rural. Y además que podría solucionar con cambios estructurales en el sistema de la tenencia de la tierra (BECKER, 2012) ${ }^{12}$.

El racismo, articulado consustancialmente al colonialismo como proceso histórico, constituyó una forma de violencia global que interrumpió, invadió y desgarró el curso de las historias mapuches. Las conse- 
cuencias del accionar colonial se pueden ver en varios ámbitos. Por ejemplo, por mencionar algunos: las tierras de nuestros antepasados han sido ocupadas de forma masiva como sustento económico para la República chilena, a partir de un modelo extractivista que hoy causa graves dańos ecológicos... como parte de un plan de mayor envergadura para conquistar y asimilar a nuestros antepasados a la fuerza a la "chilenidad". Sin desconocer el interesante debate que se ha abierto - el cual profundiza los antecedentes y complejiza la historia de la colonización - , nos preocupa que nuevamente las ciencias sociales sean utilizadas como intentos por deslegitimar la lucha política de nuestra gente (ALVARADO LINCOPI et al., 2015, p. 1).

Una coyuntura reciente y relevante ocurre en Bolivia, en la década pasada. En 2005, con la elección presidencial de Evo Morales, las identidades indígenas pasan a utilizarse como mediaciones estratégicas entre clase social y etnia, y a la vez como sustento fundamental de la acción política pública, que se puede conceptualizar como una auténtica lucha de clases desde la subalternidad etnolingüística. En efecto, los movimientos indígenas en Bolivia y otros países latinoamericanos acceden, diferenciadamente, a espacios políticos nacionales, debido a que sus movilizaciones, reivindicaciones y demandas políticas y ciudadanas incorporan discursos de identificación étnica y de pertinencia cultural (PAJUELO, 2007).

Las discusiones sobre el carácter indígena de estos movimientos políticos - por ejemplo, la controversia sobre si el mayor "beneficio y potenciación" proviene de la ideología de clase social o de una ideología étnica - colocan a los indígenas en espacios de poder que requieren de interpretaciones propias y de decisiones estratégicas que recaen en la educación, en derechos culturales y en políticas interculturales de lenguaje. Así, permanece abierta la discusión sobre si en la agenda indígena es más determinante la división social de clases que las adscripciones de la etnicidad o, como se pregunta Ludwig Huber (2011) en un balance sobre desigualdad, diferencia y política de reconocimiento: ¿En qué circunstancias se activan las identidades étnicas; en cuáles, las identidades regionales y, por último, bajo qué 
condiciones se ponen en práctica las determinaciones de la estructura de clases sociales?

De cualquier forma, desde esta perspectiva política de la experiencia boliviana prevalece la categoría de "etnicidad" por sobre noción de "clase social" y desde allí se emplaza a toda la estructura social, a través de acciones políticas en contra del Estado y sectores hegemónicos. Para ello, la categoría de etnicidad se desdobla en planos grupales e individuales, entre la autoidentificación cultural y lingüística y la categorización social (CUENCA, 2014). En resumidas cuentas, se trata de una estrategia de lucha contra las desigualdades, particularmente de aquellas que impiden el reconocimiento de los otros y su representación en la vida política social.

\section{LA HISTORIA INTERNA DE LOS ESTUDIOS SOCIOLINGÜÍSTICOS}

¿Qué impactos han tenido las discusiones sociopolíticas, como las mencionadas arriba, sobre la constitución científica en torno al significado comunicativo y lingüístico de la desigualdad de clases sociales y la etnodiversidad? Un primer efecto es el reconocimiento disciplinario de que existe una evidente interrelación multifactorial entre las estructuras sociales de clase, diversidades étnicas y las lenguas históricas que se expresan en variaciones lingüísticas, patrones diferenciados de comunicación y en representaciones, algunas compartidas (formatos públicos estereotipados) y regulaciones institucionales.

Aunque no se dispone aún de un modelo teórico de consenso sobre esta interrelación compleja, hay certeza de que constituye una de las claves o vectores sobre la diferenciación y el cambio social del lenguaje.

Sin mencionar los impactos que ha tenido esta discusión en otros campos de estudio del lenguaje, destaco el caso de la sociolingüística derivada de la llamada "dialectología social". En este campo, se formularon dos propuestas ("hipótesis de la diferencia" vs. "hipótesis del déficit”) que tuvieron gran difusión y aceptación a nivel global, las cuales conforman el llamado Paradigma de la discriminación (BLOMMAERT, 
2010), y que tuvieron gran difusión y aceptación a nivel global por las implicaciones cognitivas, socioculturales y educativas que suscitaba el fenómeno de la desigualdad de clases sociales y minorías etnolingüísticas en sociedades industrializadas. Haré una breve referencia a esta importante discusión sociolingüística, con el fin de obtener algunas enseñanzas para analizar las diversidades en el uso de las lenguas.

La "hipótesis de la diferencia" se difunde a partir de la obra fundacional de U. Weinreich, W. Labov y M. Herzog (1968), que introdujo la concepción de que los sistemas lingüísticos combinan su estructurabilidad con mecanismos de adaptación y evolución, mediante reglas variables y flexibilidad de normas lingüísticas, de acuerdo con los estratos socioeconómicos, estilos de habla y evaluaciones de los hablantes. La propuesta se consolida y se extiende aún más con la obra de William Labov, The Social Stratification of English of the New York City (1966), en la cual se postula que los cambios sociales del lenguaje tienen fundamentos empíricos y racionales en el sistema lingüístico.

Se inaugura de este modo una tradición correlacionista $(o$, covariacionista) para interpretar las relaciones entre factores estructurales, contextuales y evaluativos dentro de un ciclo de cambio lingüístico. La heterogeneidad de formas lingüísticas para expresar un mismo significado social tiene una expresión sistemática a través de reglas variables que permiten la existencia de normas coexistentes de uso. El mismo sistema permite transiciones o posibles evoluciones (transitions), que son el mecanismo básico y primario del cambio lingüístico.

El potencial de significado social aumenta cuando las transiciones se incorporan a patrones de correlación con variables de contextualización (imbedding), entre las cuales destacan dos recursos psicológicos y condiciones de estratificación socioeconómica. Los primeros refieren a grados de atención sobre el discurso (styles of speech), los cuales admiten una lógica de continuum progresivo desde el modo vernacular hasta el habla de declamación oratoria o lectura en voz alta de pares mínimos. Los segundos, se asocian a atributos sociométricos materiales ("clase social", "ingresos", "escolaridad") que se agrupan en categorías tales como "clase alta", "clase media" y "clase baja", entre otras. Este segundo mecanismo de cambio muestra que los estratos o sectores socioeconómicos poseen sus modelos propios de comunicación y cono- 
cen también modelos ajenos de referencia o contraste, lo que genera una dispersión cotidiana de modelos de lenguaje.

El tercer mecanismo de cambio (evaluations) consiste en factores psicosociales ideológicos o identitarios que fijan la dirección de los cambios por medio de correlaciones o parámetros de dominación y status. De este modo, la estratificación socioeconómica constituye el mayor factor de ordenamiento y jerarquización de la variabilidad lingüística.

En su tiempo, este modelo covariacionista (transición-contextualización-evaluación) rivalizó con la teoría de la acomodación del habla (Speech Accommodation Theory), liderada por H. Giles y P. Powesland (1997), que cuestionaron el paradigma laboviano, especialmente las correlaciones con los estilos y estratos socioeconómicos y se enfocaron sobre los procesos sociales cognitivos que expresan las percepciones individuales de los hablantes sobre el contexto y los comportamientos comunicativos.

The essence of the theory of accommodation lies in the social psychological research on similarity-attraction. This work suggests that an individual can induce another to evaluate him more favourably by reducing dissimilarities between them. The process of speech Accommodation operates on this principle and as such may be a reflection of an individual's desire for social approval (GILES; POWESLAND, 1997, p. 235).

En esta teoría se intenta demostrar el valor de los procesos psicosociales de convergencia y divergencia para comprender la dinámica de la diversidad de hablas en los contextos sociales, independientemente de las clases sociales. Pero coinciden con Labov en que el dispositivo ideológico tiene una gravitación estratégica para determinar el perfil sociohistórico de las hablas y de los discursos.

Otra propuesta interesante que también muestra compatibilidad con el sistema de ideas de Labov, en cuanto a la determinación ideológica de los procesos sociales comunicativos es la teoría de las redes 
sociales como normatividad (funcional y de diferenciación discursiva) de L. Milroy (1991, p. 9).

En los ańos setenta, por otra parte, se convirtió en una referencia clásica la discusión sobre los códigos sociolingüísticos, que introdujo B. Bernstein (1971), a propósito de los aprendizajes escolares.

Es indudable, que la clase social determina muy profundamente las formas de socialización. La estructura de clases influye en el trabajo y en los roles educativos, controla las relaciones que las familias pueden tener entre ellas y penetra de manera profunda la experiencia social inicial, adquirida en la familia. El sistema de clases influye poderosamente en la distribución del conocimiento entre los miembros de una sociedad; también condiciona el sentimiento que les permite actuar sobre el mundo, el cual es compartido de manera desigual. El sistema de clases separa claramente las comunidades unas de otras jerarquizándolas y oponiéndolas sobre una envidiosa escala de valores. La influencia de la clase social se inscribe, por consiguiente, en tres dimensiones fundamentales: el conocimiento, la posibilidad de actuación, y la distancia u oposición entre los grupos (BERNSTEIN, 1971, p. 5).

Según Bernstein (1971), es evidente que las diferencias entre clases sociales que viven en muy diferentes condiciones materiales ejercen influencia sobre las formas de control y de innovación de procedimientos de socialización. En consecuencia, la estructura profunda de la comunicación experimenta transformaciones, aunque no de un modo irrevocable y definitivo.

Para contrarrestar el controvertido procedimiento de las correlaciones, surge desde la tradición romanística alemana el modelo de condicionamiento recíproco desde dos direcciones (SCHLIEBEN-LANGE, 1977). Una dirección es el condicionamiento social de las lenguas, en dos sentidos. Por un lado, todas las lenguas se manifiestan siempre como lenguas individuales históricas y, por eso, su existencia está asociada a una determinada sociedad, clase social, estrato 
socioeconómico, nación o minoría, cosa bastante visualizada en América Latina con las comunidades indígenas. Pero, además, las estructuras sociales de relevancia o sectores sociales en emergencia crean necesidades denotativas para la lengua o lenguas disponibles en una comunidad.

Se vuelve pertinente, en este sentido, la crítica de las categorías estructuralistas establecidas, tanto sociales como lingüísticas. Para comenzar están las nociones de "sociedad de clase" y "diferencias de clase" en el lenguaje. Es frecuente escuchar expresiones tales como: "se nota su origen de clase cuando habla" o "la clase alta domina en los medios de comunicación" para postular conclusiones sobre los particularismos de la comunicación lingüística en determinadas sociedades. En suma, asumir que las llamadas clases sociales viven de acuerdo con un conjunto homogéneo de creencias y valores culturales resulta siempre una simplificación extrema.

Hay que considerar, por otra parte, que existe una diferencia entre el uso cotidiano y la terminología técnica y académica. Aunque, en la búsqueda de una definición de este término, los investigadores en sociolingüística no tienen un acuerdo sobre una definición operativa que sea apropiada para todo tipo de estudio sobre las diferencias sociales en el lenguaje. Emplear la expresión "clase social en el lenguaje” como una categoría de análisis, es decir, como una dimensión a través de la cual se expliquen procesos sociales y, en particular, para sustentar acciones autogestionarias sobre la lengua, la cultura y otros bienes es algo sumamente difícil de justificar. Sinceramente, ¿deseamos que este uso de "clase social en el lenguaje" permanezca abierto a este tipo de interpretaciones o manipulaciones?

La segunda dirección del condicionamiento recíproco es el condicionamiento lingüístico de la sociedad. La lengua crea identidad. Las naciones y — quizás aún en mayor medida, las subculturas — se disgregan del mundo exterior mediante lenguas comunes, que refuerzan su cohesión in-group (SCHLIEBEN-LANGE, 1977).

Cualquier análisis de la diferenciación social del lenguaje debería basarse en una teoría general de la práctica social (performance, actuación), que defina la relación entre acciones no lingüísticas y lingüísticas en marcos culturales y que además replantee las dimensiones de diferenciación de los sistemas de actuación. Una teoría semejante no se ha formulado hasta 
ahora; por eso no podemos referirnos explícitamente a una teoría sobre las relaciones entre lenguas y estructuras de clases sociales (GUMPERZ, 1996; SCHLIEBEN-LANGE, 1977; LUCHMANN, 1969).

Un aspecto central que resta por aclarar es la función de la ideología: se establecen estratos o clases también en la conciencia de los hablantes, a partir de la división social del trabajo: tipos de trabajo (manual/planificador), diferencias de ingresos y escolaridad, lo cual muchas veces impide la solidaridad de clases $^{13}$. Pero si estamos interesados en obtener conclusiones acerca de las acciones humanas, un enfoque de la interrelación "lengua-clase social" seguramente prejuiciará nuestro análisis, del mismo modo en que un estudio guiado por categorías sociales no explicadas resulta muy cuestionable. Las estructuras sociales, como todos lo sabemos, están marcadas por grandes diferencias en sus creencias y valores morales y políticos. Estudios antropológicos y de política del lenguaje que emplean el factor de las ideologías lingüísticas proporcionan mucha evidencia al respecto.

No menos importante, desde el punto de vista de la evolución interna de la disciplina, es cierto nivel de frustración por los pocos resultados del énfasis evidente que se puso en el conocimiento teórico-empírico del desarrollo sociopolítico y sociocultural de las comunidades de habla y una aguda conciencia o reflexión sobre la metodología, a raíz de las insatisfacciones por la extrema "ascesis" de la lingüística del siglo XX. A mediados de los años 60 tenían vigencia por doquier las mismas premisas metodológicas de transformación, solidaridad y liberación de las jerarquías sociolingüísticas y culturales. Sin embargo, puede afirmarse sin exagerar que la alusión a la heterogeneidad sistemática, a la estratificación social fundamental y a las socializaciones múltiples en los contextos comunicativos e interacciones de actuación no llegó a formar escuela, ni mucho menos un nuevo paradigma de los estudios funcionales e históricos del lenguaje (FISHMAN, 1968; FISHMAN; GARCÍA, 2011).

Ha sido E. Coseriu (1962), según B. Schlieben-Lange, el teórico que vio mejor la dificultad del estructuralismo para acotar en términos de análisis la heterogeneidad de una determinada lengua histórica. Coseriu (1962) distingue dos planos: lengua funcional, que es homogéneo, donde funcionan las mismas oposiciones estructurales, que obedece a necesidades de descripción. Y un segundo plano: la "lengua histórica concreta”, que revela la arquitectura de la lengua. 
Para tipificar la arquitectura de la lengua histórica, Coseriu (1962) propone un modelo de cuatro dimensiones de diferenciación (diacronía, diatopía, diafasía y diastratía) que no están en secuencia progresiva como el modelo de Labov y que no desarrolla ninguna base sociológico o cultural para diferenciar los estratos socioeconómicos como factor de significación social.

En síntesis, las investigaciones ejemplares en sociolingüística que aluden a las estructuras de diferenciación de estratos o clases sociales no incorporan elementos de las discusiones de economía política o de sociología de la división social del trabajo o sistemas de creencias sobre dominación y jerarquías socioculturales. Más bien, las aludidas propuestas consideran las estructuras de clases sociales como categorías descriptivas del cambio en las formas y uso lingüístico. Esto es, una aplicación metodológica.

Hemos llegado, finalmente, a un punto en que tenemos que abandonar el intento de situar a la desigualdad de clases en el lenguaje en una sola esfera disciplinaria e intensificar la crítica por omisión en las discusiones estratégicas de la lingüística social contemporánea, entre las cuales tienen gran relevancia las regulaciones más bien jurídicas y escolares en políticas del lenguaje. No es la mejor solución ignorar la historia de estratificaciones, desigualdades de clases y las relaciones culturales globales en la organización comunicativa de las sociedades específicas, y modelizar la influencia de la desigualdad de clases sociales y las diversidades étnicas en el lenguaje mediante modelos analíticos basados en taxonomías discretas y representaciones relativistas bipolares del espectro sociopolítico de los hablantes. Al menos, corresponde reinstalar este fenómeno en el programa científico de la sociolingüística proclive a los pueblos indoamericanos y retomar las potencialidades que proporcionan los análisis de dominación desde etnicidades emergentes.

\section{CONCLUSIÓN}

La apreciación global del significado de las clases sociales y de la etnodiversidad en el lenguaje se sustenta en enfoques de democratización y dignificación de identidades, pero el solo reconocimiento de la diferencia- 
lidad y validez de discursos y variantes lingüísticas no detienen ni revierten las prácticas de dominación y de jerarquización en el uso de idiomas y relaciones socioculturales, como indicios de la desigualdad de clases sociales. Bajo esta percepción, los significados contemporáneos de las estratificaciones sociales del lenguaje resultan ser plenamente compatibles con la pérdida de idiomas, de la distribución desigual del saber social y de la transición de los circuitos de diversidad local, nacional y global hacia parámetros de uniformidad lingüística (CALVET, 2004; BASTARDAS I BOADA, 2002).

La estructura social de clases y la etnodiversidad actúan sobre la estructura profunda de la comunicación en el curso de las diversas socializaciones que determinan la trayectoria social de los hablantes. Si bien estas configuraciones no se reducen a los aspectos lingüísticos, tampoco existe un modelo teórico de consenso que defina estas determinaciones. La realización de este tipo de análisis permite comprender la naturaleza comunicativa de los procesos sociales, la forma como estratos, grupos y clases se delimitan y cómo opera, a modo trasfondo principal del asunto, el fenómeno de la dominación simbólica en sociedades complejas modernas, donde coexisten diversas estructuras sociales, no sólo las de clases sociales.

En este período de complejas transiciones de cambios políticos y socioculturales surgirán conflictos, vacíos de investigación y barreras de distinto tipo en los marcos legales, institucionales y comunitarios para aceptar nuevos discursos y prácticas interculturales de reconocimiento de clases sociales, en lo que se refiere a usufructo de servicios, instituciones y patrimonios. Cabe, en todo caso, la duda si en la ideología de los ciudadanos multiculturales entrará como un compromiso sincero con este tipo de reorganización liberadora y que no se asumirá como otro de los ya numerosos significantes ambiguos que mueven los discursos públicos.

Toda gestión sobre el lenguaje requiere de una comprensión precisa del multilingüismo y de la estructura de la sociedad, así como del espacio social y demográfico multidimensional. De cualquier forma, las propuestas democratizadoras deberán armonizar el desarrollo de competencias y repertorios comunicativos con la libertad y la integración democrática de los diversos estratos y clases sociales.

Finalmente, en el mundo súper diverso del siglo XXI, los seres humanos viven sus vidas en un mundo reticular de apertura y complejas 
interacciones que a menudo implican desarrollar vínculos existenciales significativos a más de un país de origen, un área de residencia, un sitio de trabajo y educación escolar, rebasando la correlación entre el espacio social-simbólico con el espacio socioeconómico, cuestionando supuestos muy arraigados sobre pertenencia, desarrollo y equidad (BLOMMAERT, 2013).

\section{REFERENCIAS}

ALVARADO LINCOPI, C. et al. El colonialismo de izquierda. CHM, 2015. Disponible en: <https://www.comunidadhistoriamapuche.cl/el-colonialismode-izquierda/>. Acceso en: 30 ago. 2017.

BALTHASER, B. Marxismo y luchas indígenas. Marxismo Crítico, 2017. Disponible en: < https://marxismocritico.com/2017/06/23/marxismo-y-luchasindigenas/>. Acceso en: 23 ago. 2017.

BASTARDAS I BOADA, A. Política lingüística mundial a l'era de la globalització: diversitat i intercomunicació des de la perspectiva de la "complexitat". Noves SL, México, v. 1, p. 1-14, 2002. Disponible en: <https://www.researchgate. net/publication/277276750 Politica linguistica mundial a l'era de la globalitzacio diversitat i intercomunicacio des de la perspectiva de la 'complexitat'>. Acceso en: 2 sep. 2017.

BECKER, M. Mariátegui y el problema de las razas en América Latina. Revista Andina, Cuzco, n. 35, 2012. Disponible en: <https://www.yachana.org/ research/revistaandina.pdf>. Acceso en: 2 sep. 2017.

BERNSTEIN, B. Clases sociales, lenguaje y socialización. Class, Codes and Control. Londres/Nueva York: Routledge \& Kegan Paul, 1971. v. 1. Disponible en: <http:// www.infoamerica.org/documentos pdf/bernstein05.pdf $>$. Acceso en: 2 sep. 2017.

BLOMMAERT, J. Ethnography, Superdiversity and Linguistic Landscapes. Chronicles of Complexity. Bristol, Buffalo, Toronto: Multilingual Matters, 2013.

BLOMMAERT, J. The Sociolinguistics of Globalization. Cambridge: Cambridge University Press, 2010.

BYRD, J.A. Mind the Gap: Indigenous Sovereignty and the Antinomies of Empire. In: LUISETTI, F.; PICKLES, J.; KAISER, W. (orgs.). The Anomie of the Earth: Philosophy, Politics, and Autonomy in Europe and the Americas. Durham: Duke University Press, 2015. p. 110-136. 
CALVET, L.-J. Globalización, lenguas y políticas lingüísticas. In: INSTITUTO CHILENO-FRANCÉS. La diversidad cultural, un debate en Chile. Santiago: Le Monde Diplomatique, Instituto Chileno-Francés/Creemos en los Sueños, 2004. p. 39-54.

COSERIU, E. Teoría del lenguaje y lingüistica general. Madrid: Gredos, 1962.

CRYSTAL, D. Language Death. Cambridge: University Press, 2000.

CUENCA, R. Historias, trayectorias y contextos: la formación de profesionales indígenas en Bolivia y Perú. In: CUENCA, R. (org.). Etnicidades en construcción. Identidad y acción social en contextos de desigualdad. Lima: IEP, 2014. p. 167-216.

DORTIER, J-F. L'homme cet étrange animal... Aux origines du langage, de la culture et de la pensée. Auxerre: Sciences Humaines, 2004.

DOWNES, W. Language and Society. Cambridge: University Press, 1998.

FISHMAN, J. Readings in the sociology of language. The Hague. Paris: Mouton, 1968.

FISHMAN, J.; GARCÍA, O. Handbook of Language and ethnic Identity. Ther Success-Failure Continuum in Languagee and Ethnic Identity Efforts. Oxford: Oxford University Press, 2011.

GILES, H.; POWESLAND, P. Accommodation theory. In: COUPLAND, N.; JAWARSKI, A. (orgs.). Sociolinguistics: a reader and coursebook. Nueva York: MacMillan Press, 1997. p. 232-239.

GUMPERZ, J. El significado de la diversidad lingüística y cultural en un contexto post-moderno. In: MUÑOZ CRUZ, H.; LEWIN, P. (orgs.). El significado de la diversidad lingüistica y cultural. México: Universidad Autónoma Metropolitana-Iztapalapa/Centro INAH Oaxaca, 1996. p. 33-47.

HAMEL, R.; MUÑOZ, H. La sociolingüística en América Latina: notas sobre su dependencia y perspectivas. In: LASTRA, Y.; HAMEL, R.; MUÑOZ, H. (orgs.). Sociolingüística latinoamericana. México: Instituto de Investigaciones Antropológicas de la UNAM, 1988. p. 227-240.

HOUSE, N.H.; KLOESEL, C. The Essential Peirce, Selected Philosophical Writings, (1867-1893). Bloomington: University Press, 1992.

HUBER, L. Desigualdad, diferencia y "políticas de la identidad". In: COTLER, J.; CUENCA, R. (orgs.). Las desigualdades en el Perú: balances críticos. Lima: Instituto de Estudios Peruanos, 2011. p. 97-132. 
LABOV, W. The Social Stratification of English of the New York City. Washington D.C.: Center for Applied Linguistics, 1966.

LAFONT, R. La spectacularisation de l'occitanophonie dans l'enquête sociolinguistique: la fonction du "retour". Lengas, Revue de Sociolinguistique, 7, p. $71-77,1980$.

LUCHMANN, T. Soziologie der Sprache. In: KÖNIG, R. (org.). Handbuch der Empirischen Sozialforschung II. Stuttgart: Ferdinand Enke, 1969. p. 1050-1101.

MAR-MOLINERO, C.; STEVENSON, P. Language ideologies, policies and practices: language and the future of Europe. Hampshire: Palgrave MacMillan, 2006.

MILROY, L.. Language and Social Networks. 2. ed. Oxford-New York: WileyBlackwell, 1991.

MUÑOZ CRUZ, H. Reflexividad sociolingüistica de hablantes de lenguas indígenas. Concepciones y cambio sociocultural. México: Universidad Autónoma Metropolitana-Iztapalapa, 2010.

PAJUELO, R. Reinventando comunidades imaginadas: movimientos indígenas, nación y procesos sociopolíticos en los países andinos. Lima: Instituto Francés de Estudios Andinos/Instituto de Estudios Peruanos, 2007.

PEIRCE, C. S. The essential Peirce. Bloomington: Indiana University Press, $1992.2 \mathrm{v}$.

PUTNAM, H. Mind, Language and Reality. Philosophical Papers. Cambridge: University Press, 1975.

ROJAS, J. M. Estructura social, poder y clases sociales. Departamento de Ciencias Sociales, 1992. Disponible en: <http://bibliotecavirtual.clacso.org.ar/ar/libros/ colombia/cidse/doc21>. Acceso en: 14 mayo 2018.

SCHLIEBEN-LANGE, B. Iniciación a la sociolingüistica. Madrid: Gredos, 1977. SPERBER, D. Explicar la cultura. Un enfoque naturalista. Madrid: Morata, 2005. WEINREICH, U.; LABOV, W.; HERZOG, W. Empirical foundations for a Theory of language change. In: LEHMAN, W.P.; MALKIEL, Y (orgs.). Directions for historical linguistics. Austin, Texas y Londres: University of Texas Press, 1968.

WITTGENSTEIN, L. Tractatus logico-philosophicus-Investigaciones filosóficas. Madrid: Gredos, 2017. 


\section{NOTAS}

1. Los estudios de los hechos y procesos sociolingüísticos de los contextos locales, regionales o nacionales rara vez trascienden las fronteras del país respectivo (HAMEL; MUÑOZ, 1988).

2. Razones políticas y reformas educativas inspiradas en la industrialización y modernización para insertar a comunidades y pueblos minorizados como reservas laborales en los años 60-70 favorecieron la aceptación y la confianza en la sociolingüística.

3. Un aspecto destacable en la historia externa de la sociolingüística es que por casi sesenta años de trayectoria disciplinaria ha mantenido una vocación solidaria y reivindicativa a favor de comunidades etnolingüísticas vulnerables y minorizadas, ratificando la ruptura con tendencia ascética de la lingüística estructuralista y transformacional del Siglo XX (SCHLIEBEN-LANGE, 1977), que intentaban mantener en un sitio irreductible la homogeneidad de las lenguas y la estabilidad de los sistemas lingüísticos como principios de interpretación.

4. A pesar de la gran cantidad de nueva e invaluable información descriptiva de las últimas décadas, y de los nuevos conocimientos adquiridos, está lejano todavía el desarrollo de un conjunto básico de premisas teóricas, a partir de las cuales se creen consensos sobre método e información relevante sobre los procesos sociales en el lenguaje.

5. La sociolingüística interaccional es una forma de lingüística y del análisis del discurso. El objeto de estudio lingüístico no son las frases o las cláusulas, sino prácticas comunicativas propias del uso cotidiano del lenguaje. Es claro que hablar de análisis del discurso significa algo muy distinto al análisis de las frases, ni el discurso es dialógico por naturaleza. Los encuentros reales implican un orden sistemático (GUMPERZ, 1996).

6. Trad. Cuando introdujimos variables sociolingüísticas, se relacionó principalmente con los dos factores sociales interconectados de clase y estilo. Esto es importante, pero en cierto modo engañoso, ya que sólo ilustra una estructura sociolingüística particular... Los principales factores son: (1) el espacio geográfico; (2) estratificación; (3) redes sociales; (4) sexo; (5) etnicidad; (6) edad. Estos se tejen de una manera muy sutil y compleja en una comunidad de habla (DOWNES, 1998, p. 176).

7. La teoría de los signos de Peirce (1992) es esencialmente una teoría social. Especialmente, si se abandona la concepción de las representaciones mentales ubicadas en la mente de los individuos y más bien las concebimos como procesos socialmente compartidos. Este es un argumento bien conocido dentro de la filosofía, particularmente en una etapa de la obra de Wittgenstein (2017).

8. Existe una suerte de subordinación teórico-metodológica y temática de esta disciplina para actualizar, recrear y divulgar la nueva información científica sobre este campo disciplinario, lo que refleja las limitaciones de las instituciones e investigadores latinoamericanos para producir su propia acumulación científica. 
9. Mientras que muchos sindicatos han emitido declaraciones de apoyo a la lucha de Standing Rock de los sioux en defensa de sus tierras y su tratado, el hecho de que la confederación sindical más importante de EEUU (AFL-CIO) respalde la construcción del oleoducto indica que todavía existe una brecha entre las luchas de los pueblos indígenas y las de los trabajadores. El "desarrollo" convierte a las personas y la tierra en insumos abstractos (BALTHASER, 2017).

10. Complementariamente al enfoque sobre la raza, se destaca en estas discusiones el interés por definir lo específico de la lucha indígena, agregando la problemática de la justicia ecológica. La lucha por la soberanía indígena no sería contradictoria con el deseo de transformar la modernidad capitalista, sino que es un elemento central del mismo.

11. En esos años, abogaba por el establecimiento de unas "repúblicas nativas independientes" para los negros en Sudáfrica y los Estados Unidos. Estas discusiones sobre el papel de la raza en los movimientos revolucionarios que surgieron en Sudáfrica y los Estados Unidos pronto se extendieron a América Latina con la propuesta de crear una República Indígena entre los pueblos quechua y aymara en la región Andina (CUENCA, 2014; HUBER, 2011).

12. Mariátegui, en su obra principal, Siete ensayos de interpretación de la realidad peruana, plantea que el imperio incaico "construyó el más desarrollado y armónico sistema comunista”. La historia inca estableció una base para las soluciones comunales de los campesinos indígenas, pero se debería progresar hacia una moderna, occidental, revolución socialista en vez de intentar a regresar al pasado incaico. Para Mariátegui, sería mucho mejor que los indígenas lucharan por la igualdad dentro de la cultura dominante en vez de marginarse de los beneficios de la modernidad (apud BECKER, 2012).

13. El problema de la ideología es también objeto de algunos movimientos de la sociología, sin que este se sujete a las estructuras de clase que hay en su base. ¿qué posición ocupa la lengua en los contextos de valoración? Cabe distinguir dos aspectos: uno, los sistemas evaluativos se transmiten en términos lingüísticos. Los mismos contenidos de lengua ofrecen ciertas categorías interpretativas de la realidad preexistente. Otro, es que la lengua se convierte como totalidad en objeto de la actividad evaluativo-ideológica.

Recibido el 6 de febrero de 2019.

Aprobado el 9 septiembre de 2019.

(C) 2019 Centro de Estudos Educação e Sociedade - CEDES Este es un artículo de acceso abierto distribuido en términos de licencia creative commons. 